\title{
Enhanced anticancer activity and intracellular uptake of paclitaxel-containing solid lipid nanoparticles in multidrug-resistant breast cancer cells
}

This article was published in the following Dove Press journal:

International Journal of Nanomedicine

\section{Wenting Xu \\ Eun Ju Bae \\ Mi-Kyung Lee}

Department of Pharmaceutical Sciences, Woosuk University, Jeonbuk, South Korea
Correspondence: Mi-Kyung Lee Department of Pharmaceutical Sciences, Woosuk University, 443 Samnye-rho, Samnye-eup, Jeonbuk 55338, South Korea $\mathrm{Tel}+8263290 \quad \mid 423$

Fax $+8263290|56|$

Email leemk@woosuk.ac.kr
Purpose: The aim of this study was to show enhanced anticancer activity of paclitaxel (Ptx) incorporated into solid lipid nanoparticles (SLNs) and reveal reversal of multidrug resistance (MDR) by SLNs mediated by increased uptake through different entry mechanisms from that in drug-sensitive cells.

Methods: Anticancer activity of Ptx incorporated in SLNs (Ptx-SLNs) was measured in the drugsensitive human breast cancer cell line MCF7 and its MDR variant MCF7/ADR. Cellular uptake of cargo molecules in SLNs was compared using Ptx-SLNs and rhodamine 123-loaded SLNs (RhoSLNs) in both cell lines. In addition, endocytic uptake was evaluated using genistein (Gen) and chlorpromazine (Cpz) as inhibitors of clathrin- and caveola-mediated endocytosis, respectively.

Results: Ptx-SLNs showed remarkably enhanced anticancer activity in MCF7/ADR compared to Ptx delivered in dimethyl sulfoxide (DMSO) and Cremophor EL-based vehicles. SLNs significantly increased intracellular uptake of Ptx and Rho in MCF7/ADR. Western blotting demonstrated that clathrin was expressed in both cell lines, while caveolin 1 was expressed only in MCF7/ ADR. In MCF7/ADR, uptake of Ptx-SLNs and Rho-SLNs was reduced by Gen, while there was no change by $\mathrm{Cpz}$, suggesting the involvement of caveola-mediated endocytosis. Size reduction of Rho-SLNs through high-pressure homogenization (Rho-SLNs) appeared to cause a shift of the endocytosis mechanism from a clathrin-independent pathway to a clathrin-dependent one. In contrast to MCF7/ADR, the uptake of SLNs into MCF7 was not changed by Gen or Cpz, suggesting involvement of clathrin- and caveola-independent mechanism for the entry of SLNs.

Conclusion: MDR was reversed by incorporating drug into SLNs, and the reversal was mediated by increased uptake of SLNs evading efflux pumps in MDR cells. The enhanced uptake could also be due to the use of different endocytosis pathways by SLNs in MDR cells from drug-sensitive cancer cells.

Keywords: SLNs, MDR, paclitaxel, Pgp, breast cancer, endocytosis

\section{Introduction}

According to the American Cancer Society (ACS), breast cancer makes up 25\% of all new cancer diagnoses in women worldwide, and $14 \%$ of deaths are reported to be due to breast cancer in the US. Although mortality rates have been declining in recent decades, relapse after the first 5 years is a major cause of breast cancer mortality. ${ }^{1}$ The major reason for the relapse has been known to be multidrug resistance (MDR) acquired during chemotherapy. ${ }^{2-4}$ Therefore, overcoming MDR has been an important goal in breast cancer therapy and drug-delivery fields. 
Broadly, MDR is known to be developed by mechanisms decreasing intracellular drug concentration and altering intracellular pathways, enabling evasion from apoptosis. ${ }^{5}$ Among various specific mechanisms, expression of drug-efflux pumps has been considered one of the major reasons for MDR..$^{6-8}$ In particular, Pgp, a $170 \mathrm{kDa}$ ATP-dependent membrane efflux pump, has drawn attention for its universality in various cancers and tissues. Pgp pumps out substrate molecules that are generally hydrophobic in nature and hence partition into a lipid bilayer. For this reason, Pgp is considered a "molecular vacuum cleaner", dragging substrates from the membrane and expelling them outside cells. ${ }^{9}$ To overcome MDR, researchers have tried to inhibit Pgp-efflux using chemical inhibitors or by evading the efflux pump by incorporating drugs into nanoparticles. ${ }^{10-12}$ The expulsion of drug molecules occurs following detention of them within bilayers of cellular membrane and subsequent discharge by Pgp. Incorporation of drug molecules into nanoparticles hampers the direct interaction between drug and membrane, disguising them to enter cells using an endocytosis uptake mechanism that bypasses efflux transporters. ${ }^{13}$

Paclitaxel (Ptx) has been a widely used anticancer agent for several cancers, including breast cancer. ${ }^{14}$ Unfortunately, Ptx is a Pgp substrate and inactive in MDR cancer cells, due to the efflux mediated by Pgp. In an effort to overcome MDR in breast cancer, we loaded Ptx into solid lipid nanoparticles (SLNs) and tried to show enhanced anticancer activity in the MDR human breast cancer cell line MCF7/ADR by comparison with the drug-sensitive human breast cancer cell line MCF7. In addition, we tried to show that MDR reversal was associated with increased uptake of drug due to escape from efflux pump by SLNs and that internalization of SLNs into MDR cells was mediated by different entry pathways from those into drug-sensitive cancer cells.

\section{Methods}

\section{Materials}

Ptx, trimyristin (Tm), verapamil (Ver), genistein (Gen), chlorpromazine (Cpz), and $n$-hexyl-hydroxybenzoate were purchased from Sigma-Aldrich Co. (St Louis, MO, USA). Egg L- $\alpha$-phosphatidylcholine (PC) and DSPEmethyl(polyethylene glycol)-2000 $\left(\mathrm{mPEG}_{2,000}\right)$ were purchased from Avanti Polar Lipids (Alabaster, AL, USA). Rhodamine 123 (Rho), DAPI, Bodipy FL C 5 -lactosylceramide complexed with BSA (LacCer), human serum transferrin (hTf), Alexa Fluor 488 conjugate, PBS, and other cell-culture reagents, including RPMI 1640 media, were from Thermo Fisher Scientific (Waltham, MA, USA). Acetonitrile, tert-butyl methyl ether, and methanol were of high-performance liquid chromatography (HPLC) grade and purchased from Thermo Fisher Scientific. All other reagents were of analytical grade and used without further purification.

\section{Cell culture}

The MCF7 human breast cancer cell line and MDCK cell line were purchased from the Korean Cell Line Bank (Seoul, South Korea). The MCF7/ADR (ADR) cell line, which is an MDR variant of MCF7, was obtained from the National Cancer Institute (Rockville, MD, USA). Cells were cultured in RPMI 1640 medium containing 10\% FBS (HyClone, Logan, UT, USA), 100 units $/ \mathrm{mL}$ penicillin, and $100 \mu \mathrm{g} / \mathrm{mL}$ streptomycin at $37^{\circ} \mathrm{C}$ under $5 \% \mathrm{CO}_{2}$ environments.

\section{Preparation of SLNs}

SLNs were prepared as described in a previous study, with slight modification. ${ }^{15}$ Ptx $(5 \mathrm{mg}$ ) was dissolved in a chloroform solution containing $50 \mathrm{mg}$ egg PC and $5 \mathrm{mg}$ DSPE$\mathrm{mPEG}_{2,000}$. The chloroform solution was dried completely using nitrogen gas at $40^{\circ} \mathrm{C} . \mathrm{Tm}(100 \mathrm{mg})$ was added to a dried phospholipid mixture containing Ptx and then incubated at $65^{\circ} \mathrm{C}$ for 1 hour to allow Ptx dissolution in a melted Tm and phospholipid mixture. The hot oily mixture was dispersed in $1 \mathrm{~mL}$ prewarmed water by sonication for 5 hours at $65^{\circ} \mathrm{C}$ to obtain a milky and homogeneous dispersion using bath-type sonication (Branson Ultrasonics, Danbury, CT, USA). The resultant hot dispersion was frozen by dipping into liquid nitrogen and thawed at room temperature to make SLNs. Rho-SLNs were prepared with the same composition, except that $1 \mathrm{mg}$ Rho was incorporated instead of Ptx. In addition, a sonicated Rho-SLN dispersion was homogenized further for five cycles at $100 \mathrm{MPa}$ using high-pressure homogenization (HPH; Emulsiflex B15; Avestin, Ottawa, ON, Canada). As a preliminary experiment to measure incorporation efficiency, the resultant SLNs were subjected to Amicon Ultra 0.5 centrifugal filter unit (3 kDa cutoff; Merck, Darmstadt, Germany) at $14,000 \times g$ for 10 minutes to separate unincorporated Ptx or Rho into filtrate from SLN-associated ones. The amount of Ptx and Rho in filtrate was measured by $\mathrm{HPLC}^{15}$ and spectrofluorometry, respectively. Less than $5 \%$ of loaded Ptx or Rho was detected in the filtrate, suggesting most had incorporated in SLNs, and hence resultant SLNs were used without further separation using the centrifugal filter unit. SLN size was measured by dynamic light scattering using a Zetasizer (Malvern Instruments, Malvern, UK). All SLN dispersions were kept in a $4^{\circ} \mathrm{C}$ chamber for not more than 4 weeks until use. 


\section{Western blotting assay}

MDCK, MCF7, and MCF7/ADR cells were cultured in $75 \mathrm{~cm}^{2}$ flasks and grown to $80 \%$ confluence. After incubation, cells were washed with PBS and solubilized with ice-cold lysis buffer containing $150 \mathrm{mM} \mathrm{NaCl}, 50 \mathrm{mM}$ Tris- $\mathrm{HCl}(\mathrm{pH} 7.4), 5 \mathrm{mM}$ EDTA, $0.5 \%$ sodium deoxycholate, NP40, $10 \%$ SDS, $100 \mathrm{mM}$ phenylmethylsulfonyl fluoride, and a protease inhibitor. Insoluble materials were removed by centrifugation at $12,000 \mathrm{rpm}$ for 5 minutes. Extracted proteins were determined using a Thermo Fisher Scientific micro-BCA protein-assay kit. For caveolin and clathrin analysis, proteins were loaded onto $12 \%$ SDS-PAGE and 7.5\% SDS-PAGE, respectively, and then electrotransferred to polyvinylidene difluoride membrane. For blocking of aspecific binding, the membrane was incubated with 5\% BSA for 1 hour at room temperature. The membrane was washed three times with PBST and incubated with mouse monoclonal anticaveolin antibody and monoclonal anticlathrin antibody. After blotting with a primary antibody, the membrane was washed three times with PBST, followed by incubation with HRP-conjugated antimouse at room temperature for 1 hour. Visualization of the blots was carried out using an electrogenerated chemiluminescence-detection system.

\section{In vitro anticancer activity}

In vitro anticancer activity of Ptx-SLNs was evaluated as cell viability measured by MTT assay. MCF7 or MCF7/ ADR cells were inoculated into a 96-well plate at a density of $10^{4}$ and $0.5 \times 10^{4}$ cells/well, respectively. After incubation at $37^{\circ} \mathrm{C}$ overnight, the culture medium was replaced with fresh medium and treated with Ptx in SLNs. After 24, 48, or 72 hours, the medium containing Ptx was replaced with $180 \mu \mathrm{L}$ fresh culture medium and $20 \mu \mathrm{L}$ MTT solution $(5 \mathrm{mg} / \mathrm{mL}$ in PBS). Cells were incubated for another 3 hours, the medium removed, and $200 \mu \mathrm{L}$ dimethyl sulfoxide (DMSO) added to each well to dissolve the MTT formazan crystals. Finally, absorbance of dissolved formazan was measured after incubation for 20 minutes under agitation at room temperature at $560 \mathrm{~nm}$ with an ELISA reader (Sunrise; Tecan, Männedorf, Switzerland). Survival rates of the treated cells were calculated by comparing the absorbance with that of untreated control ones. $\mathrm{IC}_{50}$ values were calculated by nonlinear regression-curve fitting of log concentration vs percentage cell survival using GraphPad Prism 5.

\section{Intracellular uptake of Ptx in various vehicles}

MCF7 and MCF7/ADR cells were seeded in $100 \mathrm{~mm}$ plates at a density of $10^{4}$ cells $/ \mathrm{mL}$ in $10 \mathrm{~mL}$ medium and cultured for
5 days to reach $80 \%$ confluence. The medium was changed for a fresh one the night before treatment. Cells were pretreated with $20 \mu \mathrm{M}$ Ver for 30 minutes and subsequently incubated with Ptx in various vehicles at a dose of $1 \mu \mathrm{M}$ Ptx for 2 hours. After incubation, cells were washed with PBS three times, detached with trypsin, and resuspended in $1 \mathrm{~mL}$ PBS after collection by centrifugation. Cellular proteins were determined with $100 \mu \mathrm{L}$ cell suspension using the micro-BCA proteinassay kit. The rest of the cell suspension was centrifuged and the precipitated cell pellet used for analysis of Ptx taken up into cells. Methyl tert-butyl ether $(1 \mathrm{~mL})$ was added into the cell pellet with $n$-hexyl-hydroxybenzoate as internal standard and vortexed for 1 minute to extract Ptx. The upper organic layer was collected, dried using a nitrogen gas stream at $40^{\circ} \mathrm{C}$, and dissolved in $80 \mu \mathrm{L}$ mobile phase consisting of acetonitrile, methanol, and $10 \mathrm{mM}$ ammonium acetate, pH 5.0 (485:165:350 as volume ratio). Ptx was determined after injection of $50 \mu \mathrm{L}$ reconstituted extract onto a Cadenza $\mathrm{CD} \mathrm{C}_{18}$ column (ODS $3 \mu \mathrm{m}$, pore size $12 \mathrm{~nm}, 150 \times 4.6 \mathrm{~mm}$; Imtakt, Kyoto, Japan), elution with mobile phase at a flow rate of $500 \mu \mathrm{L} / \mathrm{min}$, and detection of absorbance at $227 \mathrm{~nm}$ using a Shiseido NanoSpace Si2 HPLC system. To evaluate the effect of endocytosis inhibitors, MCF7 and MCF7/ADR cells grown in $100 \mathrm{~mm}$ plates were preincubated with $200 \mu \mathrm{M}$ Gen or $10 \mu \mathrm{g} / \mathrm{mL} \mathrm{Cpz}$ for 1 hour and then treated with Ver for 30 minutes before being treated with $1 \mu \mathrm{M}$ Ptx in SLNs for 2 hours. Ptx and cellular protein in collected cell pellets after washing with PBS were determined as the aforementioned.

\section{Confocal laser-scanning microscopy (CLSM) for Rho-SLN uptake}

Cells were seeded on glass coverslips in six-well plates at a density of $3 \times 10^{5}$ cells/well and incubated overnight. Cells were treated with Rho in DMSO or SLNs at a concentration of $0.4 \mu \mathrm{M}$ Rho for 2 hours. For endocytosis inhibition, cells were pretreated with $200 \mu \mathrm{M}$ Gen or $10 \mu \mathrm{g} / \mathrm{mL}$ Cpz for 1 hour, then treated with Ver for 30 minutes prior to treatment with Rho for 2 hours. Then, cells were washed five times with PBS and fixed with $4 \%$ paraformaldehyde solution for 10 minutes at room temperature. After cells had been washed three times with PBS, cell nuclei were stained with DAPI for 5 minutes at room temperature. A drop of mounting solution was added on the center of a slide, and a coverslip was put on the small spot of mounting media with the cells down after wicking off any excess buffer. CLSM measurement and analysis were carried out using confocal microscopy (TCS SP8; Leica, Wetzlar, Germany). CLSM for uptake of endocytic markers was processed the 
same, except that cells were treated with $16.7 \mu \mathrm{g} / \mathrm{mL}$ hTf and $0.81 \mu \mathrm{M}$ LacCer for 2 hours instead of Rho-SLNs.

\section{Effect of endocytosis inhibitors on uptake of Rho-SLNs}

As a supplemental way to reveal endocytic pathways utilized by SLNs, we prepared Rho-SLNs and evaluated changes in cellular uptake of Rho depending on endocytosis inhibitors. MCF7 or MCF7/ADR cells were seeded in six-well plates at a density of $2 \times 10^{6}$ cells/well. After overnight incubation, the old medium was replaced by $1 \mathrm{~mL}$ medium containing $200 \mu \mathrm{M}$ Gen or $10 \mu \mathrm{g} / \mathrm{mL} \mathrm{Cpz}$, incubated for 1 hour, and $20 \mu \mathrm{M}$ Rho in SLNs added. After incubation for 2 hours, the medium was removed and cells washed three times with cold PBS. Washed cells were collected and cellular fluorescence measured using cytometry (Tali; Thermo Fisher Scientific).
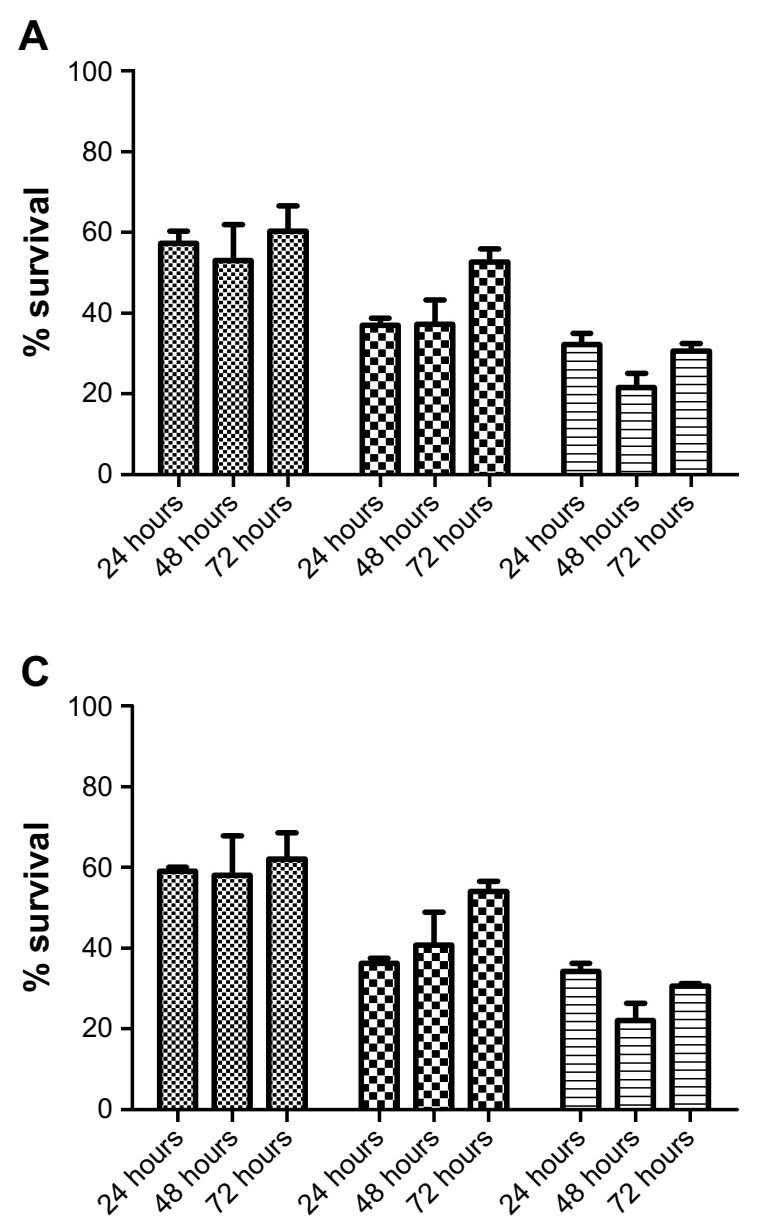

\section{Statistical analysis}

For statistical analysis, GraphPad Prism was used. Data are presented as mean $\pm \mathrm{SD}$. Comparison of two groups was performed with unpaired $t$-tests, while comparison of more than two groups was done using one-way ANOVA with Turkey's multiple-comparison test. Differences were considered significant at $P<0.05$.

\section{Results}

\section{In vitro anticancer activity of Ptx loaded in SLNs}

In the cytotoxicity assay at $1 \mu \mathrm{M}$ Ptx, 24 hour's treatment was sufficient to observe significant Ptx cytotoxicity, and prolonged treatment $>24$ hours showed no further notable increase in cytotoxicity in MCF7 cells (Figure 1A). In the case of SLNs, longer treatment for 48 hours showed higher cytotoxicity ( 24 vs 48 hours), while extending duration to
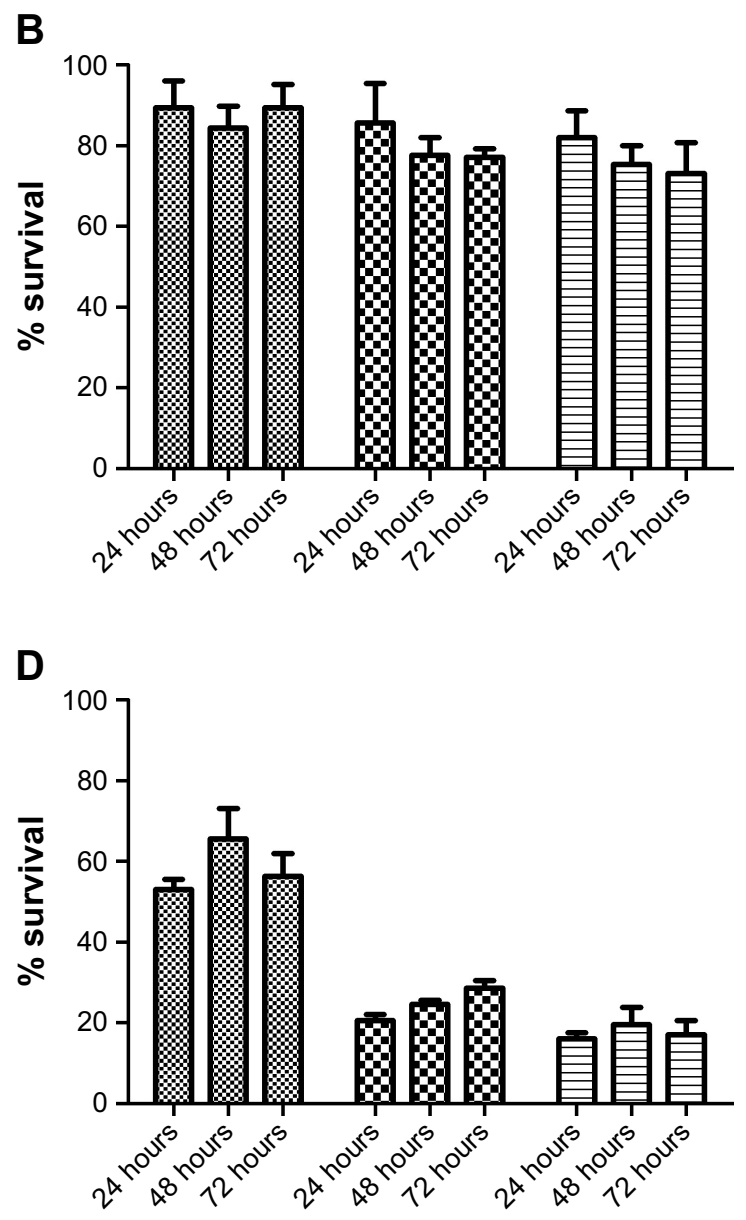

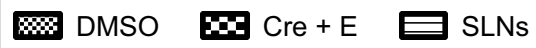

Figure I Effect of treatment duration and Ver on cytotoxicity of I $\mu$ M Ptx delivered in various vehicles in MCF7 and MCF7/ADR (ADR) cells. Notes: (A) MCF7 without Ver; (B) MCF7/ADR without Ver; (C) MCF7 with Ver; and (D) MCF7/ADR with Ver.

Abbreviations: Ver, verapamil; Ptx, paclitaxel; DMSO, dimethyl sulfoxide; Cre, Cremophor EL; E, ethanol; SLNs, solid lipid nanoparticles. 
72 hours did not show additional benefit in cytotoxicity in MCF7 cells. In MCF7/ADR cells, we did not observe any further increase in cytotoxicity from extending treatment duration (Figure 1B). Similar patterns were observed when the cells were cotreated with Ver, a Pgp inhibitor (Figure 1C and D). Overall, prolonged treatment $>24$ hours did not exhibit significant benefit in terms of cytotoxicity, which led us to treat cells for 24 hours for further experiments. In addition, cotreatment with Ver did not affect Ptx cytotoxicity in MCF7 cells that did not express Pgp (Figure 1C).

As shown in Figure $1 \mathrm{~B}$ and $\mathrm{D}$, drug resistance in MCF7/ADR was reversed by cotreatment with Ver in all vehicles. The reversal effect by Ver was stronger in Cremophor EL (Cre) + ethanol (E) and SLNs than DMSO, which proposed that there would be additional advantages in escaping Pgp-mediated efflux by Cre + E and SLN vehicles. At $1 \mu \mathrm{M}$ Ptx, there were no significant differences in cytotoxicity among vehicles. However, the superior effect of SLNs against MCF7/ADR was observed clearly in concentration-dependent cytotoxicity experiments (Figure 2 and Table 1). In MCF7 cells, Ptx in SLNs showed higher $\mathrm{IC}_{50}$ values compared to the other vehicles, regardless of cotreatment with Ver (Table 1). On the other hand, in MCF7/ADR cells, Ptx in SLNs showed remarkably improved $\mathrm{IC}_{50}$ values compared to DMSO and Cre + E vehicles. Ptx dissolved in DMSO did not exert cytotoxic effects in MCF7/ADR, an MDR cancer cell line, as expected, whereas, Cre + E vehicle increased Ptx cytotoxicity in MCF7/ADR, likely due to the Pgp-inhibitory effect of Cre. ${ }^{16}$ Based on $\mathrm{IC}_{50}$ values, SLNs were likely to evade the Pgp-efflux system in MCF7/ADR. In addition, similar $\mathrm{IC}_{50}$ values in the absence and presence of Ver suggested that Ptx loaded in SLNs was likely to be taken up in incorporated form into cells in SLNs, rather than released free form from SLNs.

\section{Cellular uptake of Ptx loaded in SLNs}

Figure 3A shows the amounts of Ptx taken up into MCF7 cells. These amounts seemed to be substantial and showed
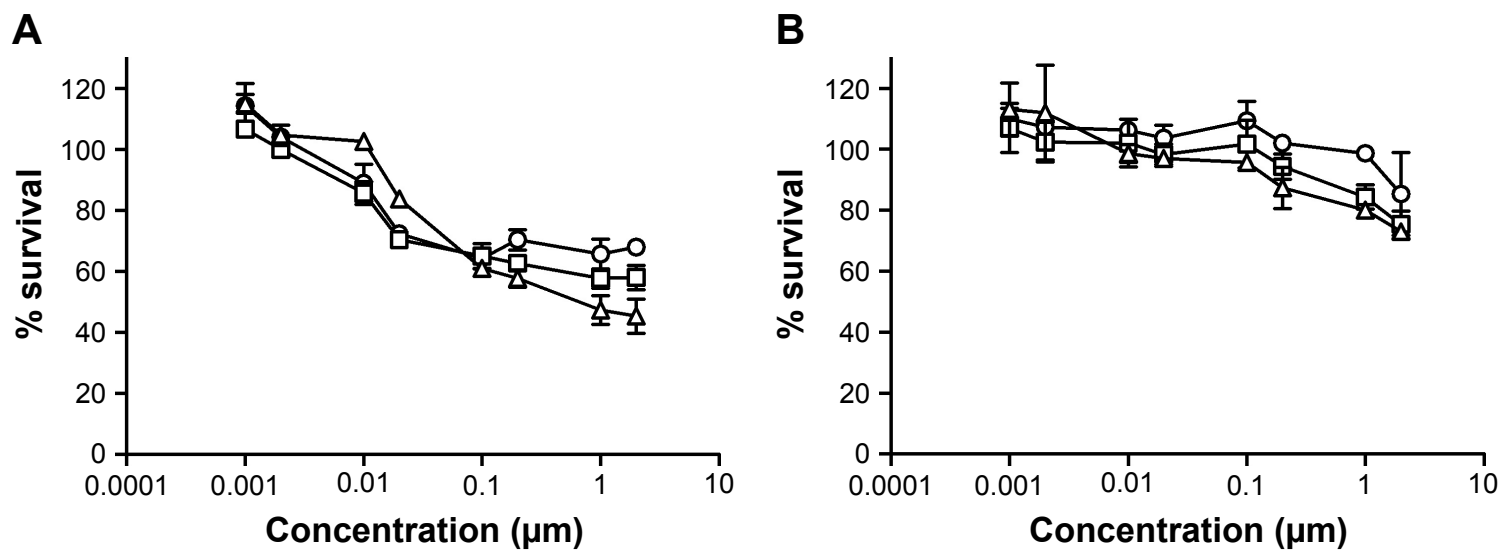

-0- DMSO $-\square$ - Cre + E $\triangle-\Delta$ SLNs
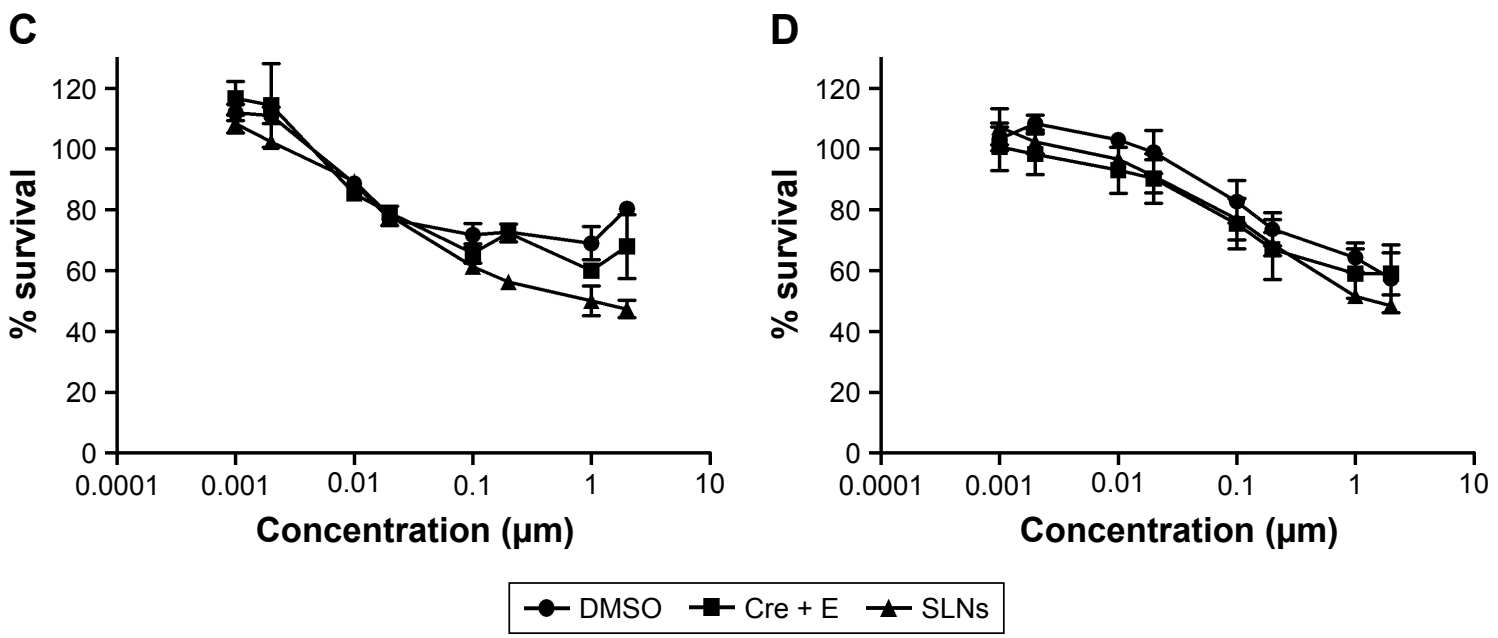

Figure 2 Concentration-dependent cytotoxicity of Ptx delivered using various vehicles in MCF7 and MCF7/ADR (ADR) cells in the absence (A and B) and presence (C and D) of Ver. Abbreviations: Ptx, paclitaxel; Ver, verapamil; DMSO, dimethyl sulfoxide; Cre, Cremophor EL; E, ethanol; SLNs, solid lipid nanoparticles. 
Table I IC ${ }_{50}(\mathrm{nM})$ values of $\mathrm{Ptx}$ loaded in various vehicles against MCF7 and MCF7/ADR with and without Ver

\begin{tabular}{|l|l|l|l|l|}
\hline \multirow{2}{*}{} & \multicolumn{3}{l|}{ MCF7 } & \multicolumn{2}{l|}{ MCF7/ADR } \\
\cline { 2 - 5 } & - Ver & + Ver & - Ver & + Ver \\
\hline DMSO & $3.6 \pm 1.0$ & $3.1 \pm 0.9$ & $>10,000$ & $88.1 \pm 24.2$ \\
Cre + E & $6.7 \pm 1.6$ & $5.4 \pm 1.2$ & $1,332.7 \pm 116.0^{\mathrm{a}}$ & $79.3 \pm 12.9$ \\
SLNs & $19.4 \pm 11.2$ & $15.5 \pm 5 . \mathrm{I}^{\mathrm{a}, \mathrm{b}}$ & $1 \mathrm{I} 2.9 \pm 44.4^{\mathrm{a}, \mathrm{b}}$ & $127.8 \pm 19.1^{\mathrm{b}}$ \\
\hline
\end{tabular}

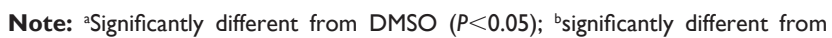
Cre + E $(P<0.05)$.

Abbreviations: Ptx, paclitaxel; Ver, verapamil; DMSO, dimethyl sulfoxide; Cre, Cremophor EL; E, ethanol; SLNs, solid lipid nanoparticles.

$40 \%-60 \%$ cell-survival rates at $1 \mu \mathrm{M}$ Ptx in various vehicles, as shown in Figure 1A. The uptake was not affected significantly by Ver in MCF7 cells, even though mean uptake values seemed to be slightly reduced by Ver in the case of Ptx in DMSO and SLN vehicles. However, negligible amounts of Ptx were taken up into MCF7/ADR cells when delivered in DMSO. Ver increased the uptake of Ptx by 2.8-fold into MCF7/ADR cells when delivered in DMSO, even though the amount was still minimal (Figure 1B). Cre $+\mathrm{E}$ also significantly increased uptake into MCF7/ ADR - approximately 23 times that of DMSO - and the effect of Ver was not as much as DMSO, as uptake was increased by only 1.2-fold compared to that in the absence of Ver. In contrast, SLNs showed remarkable increase in Ptx uptake into MCF7/ADR cells up to a comparable level of that in MCF7 cells, even though the enhanced uptake was not reflected in the cytotoxicity as much as expected (Figures 1B and 3B). Ver increased the uptake of Ptx loaded in SLNs into MCF7/ADR further by 1.3-fold compared to that without Ver (statistically significant), although the enhancement was not as much as that of DMSO and similar to that of Cre + E.

Enhancement of uptake by SLNs was also observed with Rho, which is a known Pgp substrate (Figure 4). Confocal microscopy showed that the uptake of Rho in SLNs into MCF7 cells was similar to that of Rho in DMSO. Similar to Ptx uptake, MCF7/ADR cells took up Rho much less than MCF7 cells, as shown in Figure 4. The fluorescence intensity of Rho in MCF7/ADR cells was slightly enhanced in SLNs compared to that in DMSO. Ver intensified Rho fluorescence in MCF7/ADR cells further, while it appeared not to affect that in MCF7 cells (Figure 5).

\section{Effects of endocytosis inhibitors on uptake of SLNs}

Unlike MCF7 cells, MCF7/ADR cells have been known to express Pgp, which contributes to the MDR of MCF7/ADR cells. ${ }^{17}$ Western blotting confirmed the expression of Pgp in MCF7/ADR cells used in the present study (Figure 6). In addition, MCF7 and MCF7/ADR cells exhibited different expressions of clathrin and caveolin 1, which are known to contribute to endocytosis pathways, as shown in Figure 6. Clathrin was expressed in both cell lines, whereas caveolin 1 was expressed only in MCF7/ADR cells. The different expression of clathrin and caveolin 1 in both cell lines suggested that SLNs might be taken up by MCF7 and MCF7/ADR cells using different endocytosis pathways. In further studies on SLN endocytosis, Cpz and Gen were used as inhibitors of clathrin- and caveolin-mediated endocytosis
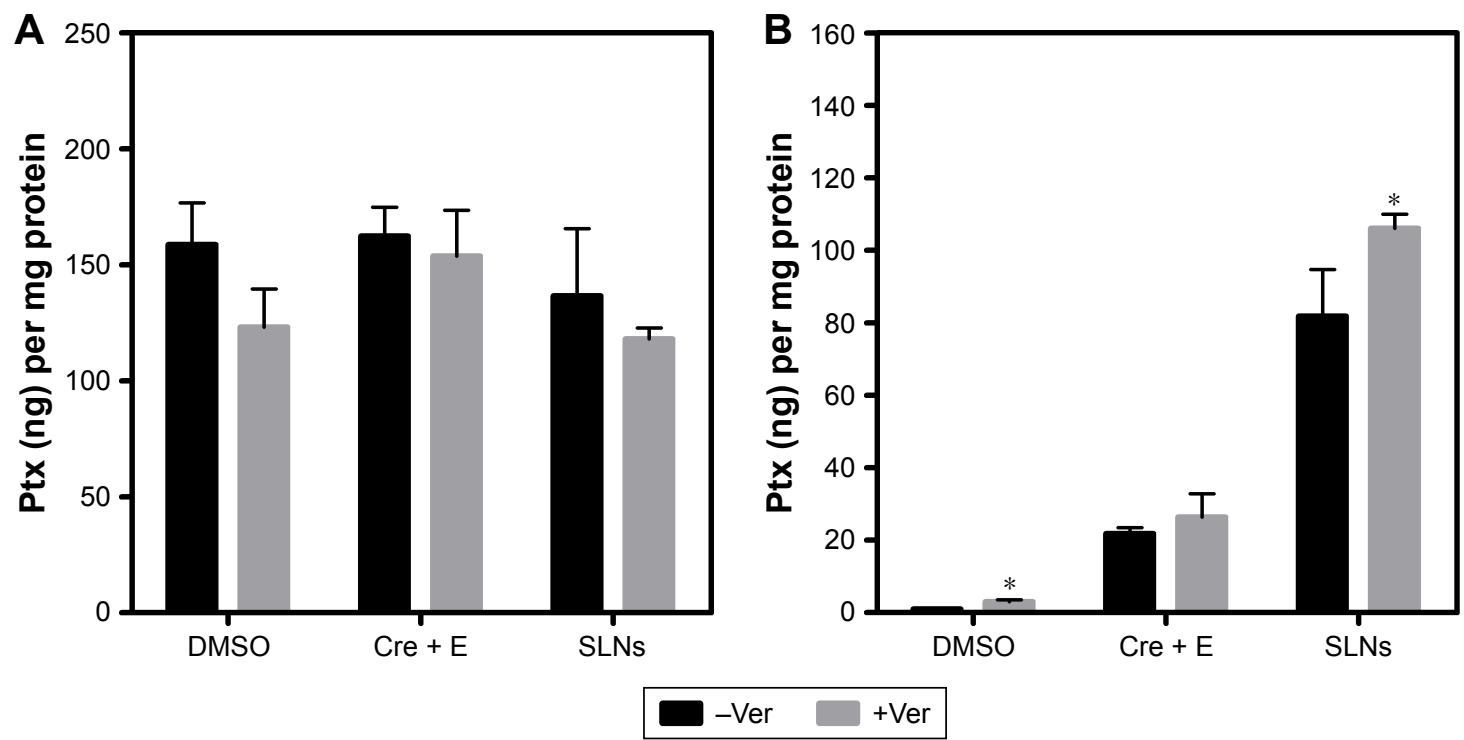

Figure 3 Intracellular uptake of Ptx delivered using various vehicles in MCF7 (A) and MCF7/ADR (B) cells in the absence or presence of Ver. Note: $*$ Significantly different compared to control $(P<0.05)$.

Abbreviations: Ptx, paclitaxel; Ver, verapamil; DMSO, dimethyl sulfoxide; Cre, Cremophor EL; E, ethanol; SLNs, solid lipid nanoparticles. 
A
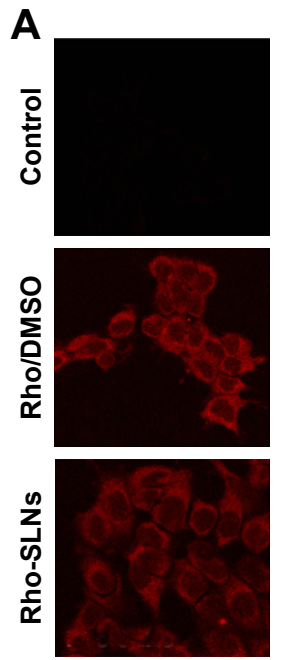
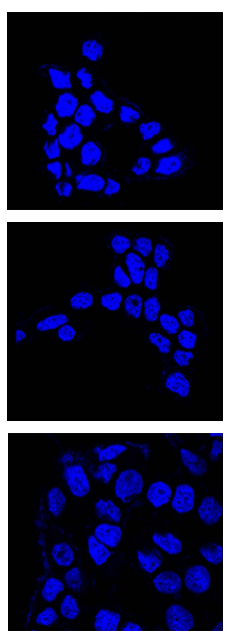
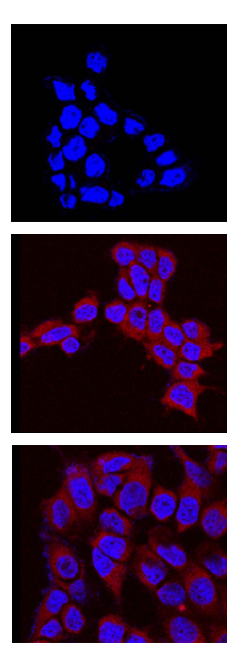

B
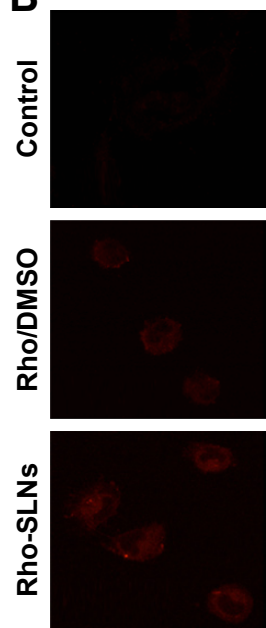
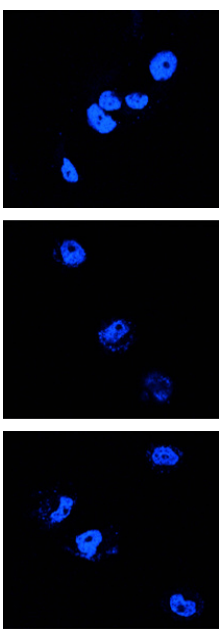
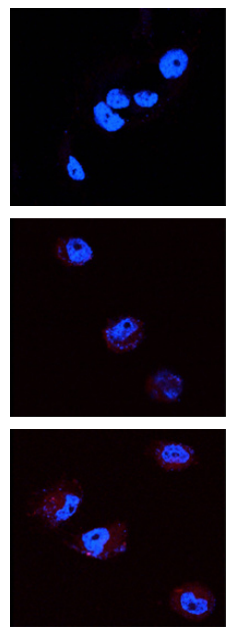

Figure 4 Confocal laser-scanning microscopy of internalization of Rho (red) in MCF7 (A) and MCF7/ADR (B) cells.

Notes: Cells were treated with $0.4 \mu \mathrm{M}$ Rho in DMSO (Rho/DMSO) or SLNs (Rho-SLNs) for 2 hours. Controls were untreated cells. Cell nuclei were stained with DAPI (blue). Abbreviations: Rho, rhodamine 123; DMSO, dimethyl sulfoxide; SLNs, solid lipid nanoparticles.

pathways, respectively. As shown in Figure 7, Gen reduced Rho uptake by $22 \%$ and Cpz increased it by $22 \%$ in MCF7/ ADR cells. However, the effects of Gen and Cpz seemed insignificant compared to that of Ver, which increased Rho uptake $\leq 244 \%$ in MCF7/ADR cells (Figure 7). For further endocytosis studies, Gen and Cpz were used concomitantly with Ver to eliminate the Pgp effect on Rho uptake.

As shown in Figure 8, lower temperature reduced PtxSLN uptake remarkably in both cell lines, indicating that energy-dependent endocytosis pathways could be involved in SLN uptake. In MCF7 cells, Gen did not change Ptx uptake, suggesting that caveolin-mediated endocytosis was not involved in SLN uptake (Figure 8). In MCF7/ADR cells, Gen reduced the uptake of Ptx loaded in SLNs by $18 \%$ compared to that without Gen $(P<0.05)$. This result appeared to indicate that caveolin-mediated endocytosis could be involved in the uptake of SLNs into MCF7/ADR, unlike with MCF7. However, the effect seemed to be too marginal to draw such a conclusion with confidence.

It was expected that Cpz would reduce Ptx-SLN uptake, because clathrin was expressed in both cell lines. However, MCF7 cells did not show significant changes in Ptx-SLN uptake following inhibition of clathrin-mediated endocytosis by $\mathrm{Cpz}$ (Figure 8). In addition, Cpz even showed a tendency to
A
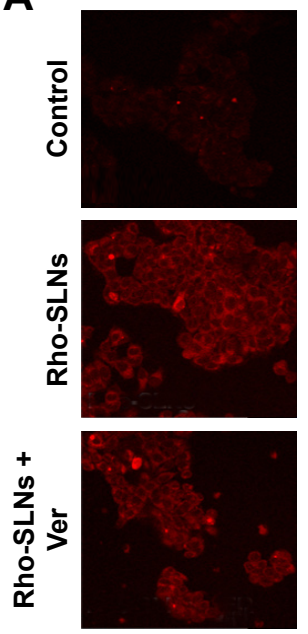
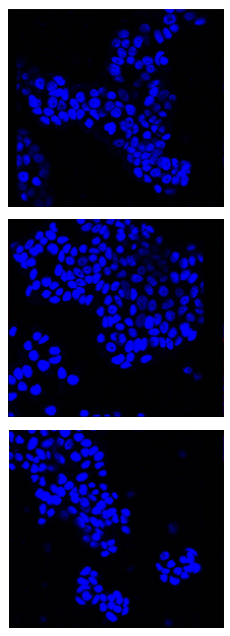
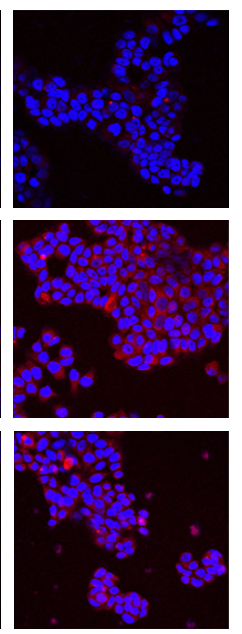

B
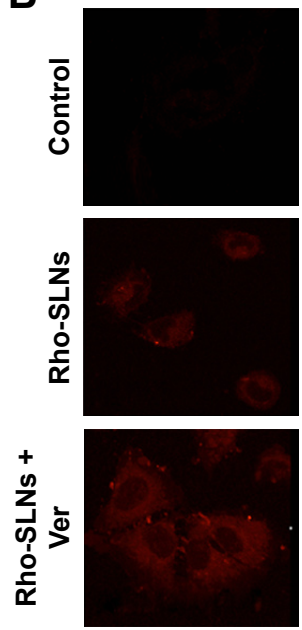
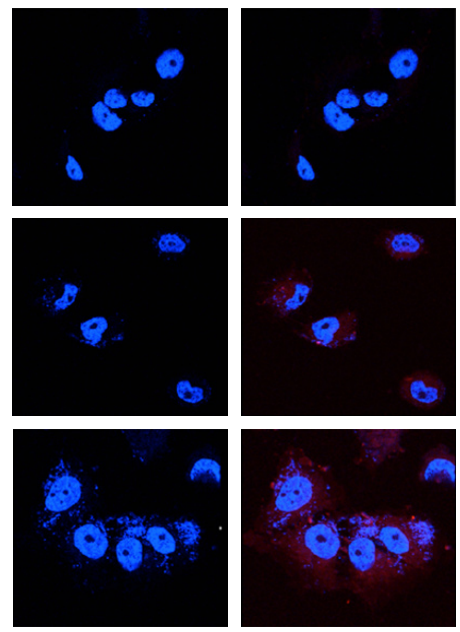

Figure 5 Effect of Ver, a Pgp inhibitor, on cellular uptake of Rho-SLNs (red) in MCF7 (A) and MCF7/ADR (B) cells.

Notes: Cells were pretreated with $20 \mu \mathrm{M}$ Ver for 30 minutes and then treated with $0.4 \mu \mathrm{M}$ Rho incorporated in SLNs (Rho-SLNs) for 2 hours. Controls were untreated cells. Cell nuclei were stained with DAPI (blue).

Abbreviations: Ver, verapamil; Rho, rhodamine 123; SLNs, solid lipid nanoparticles. 


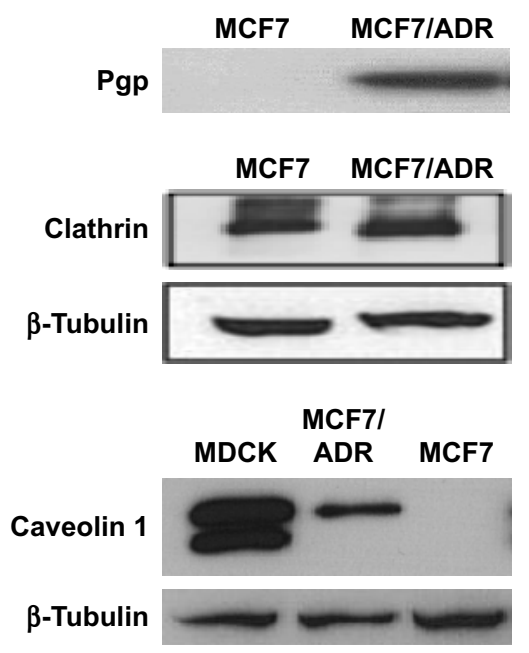

Figure 6 Western blot for expression of Pgp, clathrin, and caveolin I in MCF7 and MCF7/ADR cells.

Note: MDCK cell line was used as a positive control for caveolin I expression.

increase Ptx-SLN uptake in MCF7/ADR cells, although there was no statistical significance because of high variations.

We tried to confirm the effects of Gen and Cpz on SLN uptake using Rho instead of Ptx. Rho-SLNs and Ptx-SLNs exhibited similar mean particle size and polydispersity-index (PI) values, as shown in Table 2. Similar to the results for Ptx-SLNs, Gen and Cpz did not change the fluorescence of Rho-SLNs in MCF7 cells, while Gen reduced Rho-SLN uptake by $15 \%$ in MCF7/ADR cells (Figure 9). Cpz still caused no change in Rho-SLN uptake in MCF7/ADR cells. The uptake of SLNs into MCF7/ADR was reduced by Gen in the same manner, regardless of the incorporated agents.

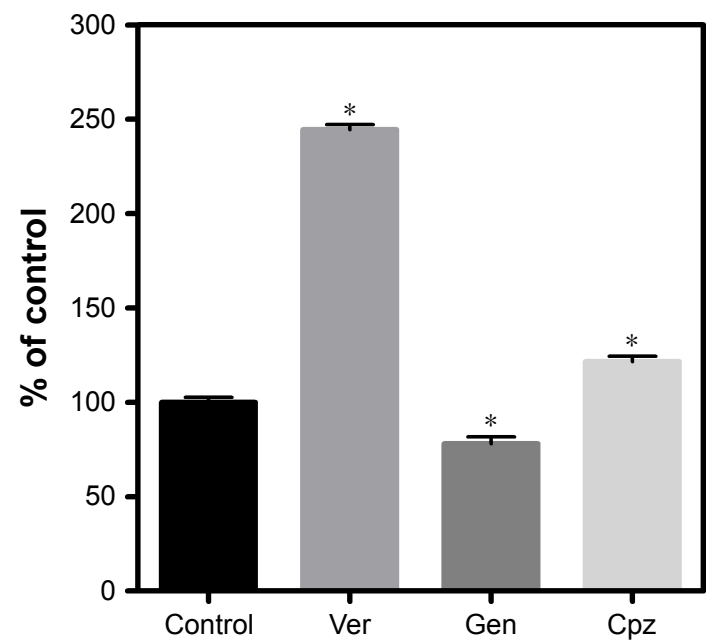

Figure 7 Effects of endocytosis inhibitors on the Pgp-mediated efflux of Rho in MCF7/ADR cells.

Notes: Intracellular Rho level expressed as percentage of fluorescence intensity compared to control cells, which were treated only with Rho in the absence of other agents. *Significantly different compared to control $(P<0.05)$.

Abbreviations: Rho, rhodamine I23; Ver, verapamil; Gen, genistein; Cpz, chlorpromazine.
However, the effect of Gen was still marginal, and this caused speculation that endocytosis pathways might be influenced by other factors, such as particle size and surface charge, and thus we could not pinpoint the effect of Gen on internalization pathways.

To address the additional effect of particle size on SLN uptake, Rho-SLN size was diminished further by HPH. The homogenized Rho-SLNs (HPH Rho-SLNs) presented much smaller mean particles and PI values compared to unhomogenized ones (Table 2). In MCF7 cells, HPH Rho-SLN uptake showed no change by Gen or Cpz, which was consistent with results for Rho-SLNs and Ptx-SLNs (Figure 10A). On the other hand, HPH Rho-SLN uptake into MCF7/ADR cells was significantly inhibited by both Gen and $\mathrm{Cpz}$, as shown in Figure 10B. The inhibited uptake in MCF7/ADR was also observed in confocal microscopy (Figure 11B). Confocal microscopy also confirmed that no change of uptake was caused by Gen and Cpz in MCF7 cells (Figure 11A).

The unchanged uptake by $\mathrm{Cpz}$ in MCF7 cells brought about a doubt that $\mathrm{Cpz}$ was an effective inhibitor for clathrinmediated endocytosis in MCF7 cells, because clathrin is known to contribute to universal endocytosis machinery, although there was a possibility that SLNs were endocytosed through routes other than clathrin-mediated pathways in MCF7 cells. Based on this doubt, we checked the effect of $\mathrm{Cpz}$ on the uptake of hTf, which has been known to enter cells using clathrin-mediated endocytosis. ${ }^{18}$ As shown in confocal microscopy images in Figure 12, the inhibition effect of $\mathrm{Cpz}$ appeared very weak in both MCF7 and MCF7/ADR cells. However, Cpz caused slight reduction in fluorescence intensity in MCF7 cells and perinuclear areas of MCF7/ADR cells (Figure 12A). In MCF7/ADR, fluorescence tended to be confined to a certain area when cells were treated with $\mathrm{Cpz}$ compared to cells without $\mathrm{Cpz}$, for which fluorescence seemed to be dispersed throughout the cytosol (Figure 12B).

Gen was also checked for its endocytosis inhibition using LacCer as a marker for caveola-mediated endocytosis. In MCF7 cells, Gen did not change LacCer uptake significantly (Figure 13A). On the contrary, in MCF7/ADR cells, the inhibition effect of Gen was significant, showing recognizable reduction in fluorescence intensity in cells, as shown in Figure 13B.

Overall, SLNs seemed to use different endocytosis pathways to enter MCF7/ADR cells from that to enter MCF7 cells. SLNs used caveola-mediated endocytosis to enter MCF7/ADR cells, even though SLNs with larger particles 
A

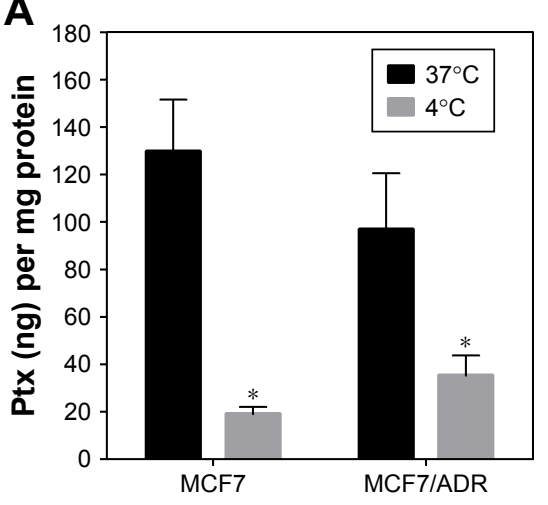

B

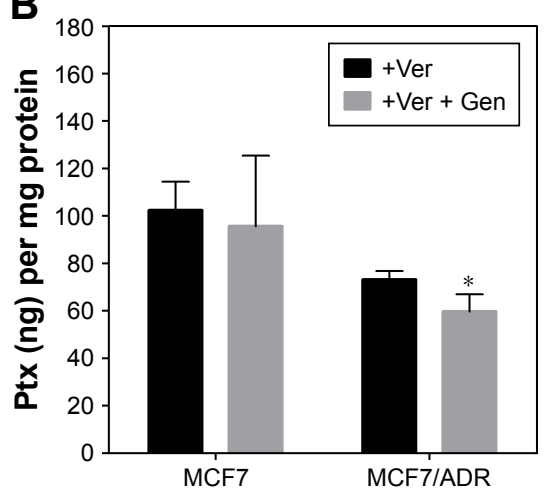

C

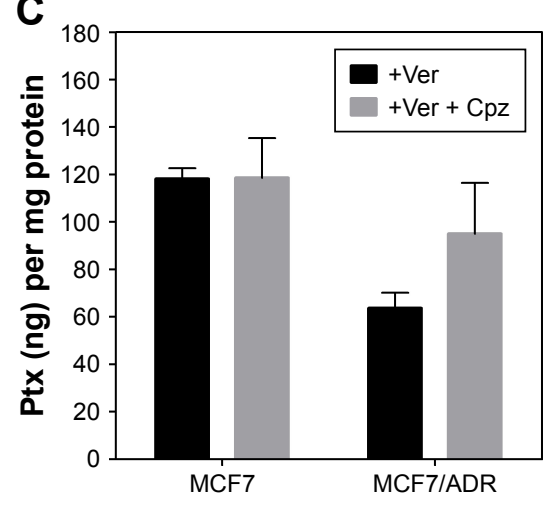

Figure 8 Effects of endocytosis inhibition by lowered temperature.

Notes: (A) $37^{\circ} \mathrm{C}$ vs $4^{\circ} \mathrm{C}$, (B) Gen, and (C) Cpz on uptake of Ptx-SLNs in MCF7 and MCF7/ADR cells. All cells were treated concomitantly with Ver to eliminate the effect of Pgp-mediated efflux on Ptx uptake. *Significantly different compared to without endocytosis inhibitor in each cell line $(P<0.05)$.

Abbreviations: Gen, genistein; Cpz, chlorpromazine; Ptx, paclitaxel; Ver, verapamil; SLNs, solid lipid nanoparticles.

appeared to use other alternative routes dominantly. In MCF7 cells, SLNs did not predominantly use either caveolamediated or clathrin-mediated pathways.

\section{Discussion}

Ptx loaded into SLNs with Tm solid core has been shown to have comparable cytotoxicity to a Cre-based formulation against ovarian and breast cancer cells in our previous study. ${ }^{15}$ In this study, a similar composition was used to prepare Tm-based SLNs, except that more egg PC and PEGylated phospholipid were utilized to stabilize SLNs compared to the previous study. ${ }^{15}$ As shown in the previous study, Ptx-free SLN vehicles did not cause significant cytotoxicity in MCF7 cells, and thus the effects of empty vehicles were not evaluated in this study. In contrast to the results in MCF7 cells, which showed comparable Ptx cytotoxicity among vehicles, Ptx-SLNs showed far more enhanced cytotoxicity compared to the Cre-based formulation in MCF7/ADR cells expressing Pgp. The Cre-based formulation also improved cytotoxicity in MDR cells compared to Ptx dissolved in DMSO, and this can be due to inhibition of Pgp by Cre. ${ }^{16}$ However, the inhibition of Pgp by Cre was likely suboptimal in the concentration range used to deliver the necessary amount of Ptx in the present study when considering $\mathrm{IC}_{50}$ values in MCF7/ADR cells were further reduced by Ver. Meanwhile, incorporation of Ptx into SLNs seemed to evade Pgp-mediated efflux fully in MCF7/ADR in consideration of remarkably enhanced $\mathrm{IC}_{50}$ values compared to free Ptx in DMSO and no further improvement in $\mathrm{IC}_{50}$ by Ver. Nanoparticles have been suggested to act as "stealth" carriers for cargos to evade discharge by Pgp-efflux pumps by impeding direct interaction of cargos with membrane and by being internalized through endocytosis in nanoparticle-associated form, rather than through diffusion across cellular membrane in free form. ${ }^{9,19,20}$ Based on the highest uptake of Ptx-SLNs into MCF7/ADR compared to Ptx in the other two vehicles, SLNs seemed to be able to enter MDR cells efficiently by bypassing efflux pumps. Consistently with our results, other researchers have also reported that SLNs reversed MDR in drug-resistant MCF7/ADR breast and SKOV3-TR30 ovarian cancer cells by increasing cellular uptake of Ptx and doxorubicin. ${ }^{21}$

Ver further increased the cellular uptake of Ptx-SLNs into MCF7/ADR by $29.7 \%$ (81.8 \pm 12.8 vs $106.1 \pm 3.8)$, suggesting that some fraction of Ptx was released extracellularly or intracellularly that resulted in its being susceptible to efflux pump, even though the amounts released appeared to be minimal due to the solidity of the SLN core, judging from results in the previous study. ${ }^{15}$

Increased uptake of cargo molecules of nanoparticles can be ascribed mainly to endocytosis-mediated Pgp circumvention and various types of endocytosis pathways can serve in

Table 2 Mean particle size and PI of SLNs prepared using sonication and HPH

\begin{tabular}{|l|l|l|l|l|l|l|l|l|}
\hline & \multicolumn{4}{l|}{ Composition of SLNs $(\mathbf{m g})$} & \multirow{2}{*}{ Particle size (nm) } & PI \\
\cline { 2 - 7 } & Tm & Egg PC & PEG $_{2,000}$ DSPE & Ptx & Rho & Dispersion method & & \\
\hline Ptx-SLNs & 100 & 50 & 5 & 5 & - & Sonication & $210.5 \pm 86.3$ & $223.6 \pm 138.2$ \\
Rho-SLNs & 100 & 50 & 5 & - & 1 & Sonication & 1.23 \\
HPH Rho-SLNs & 100 & 50 & 5 & - & 1 & Sonication + HPH & $150.8 \pm 0.2$ & 1.33 \\
\hline
\end{tabular}

Abbreviations: PI, polydispersity index; SLNs, solid lipid nanoparticles; HPH, high-pressure homogenization; Ptx, paclitaxel; Rho, rhodamine I23; Tm, trimyristin; PC, phosphatidylcholine. 
A

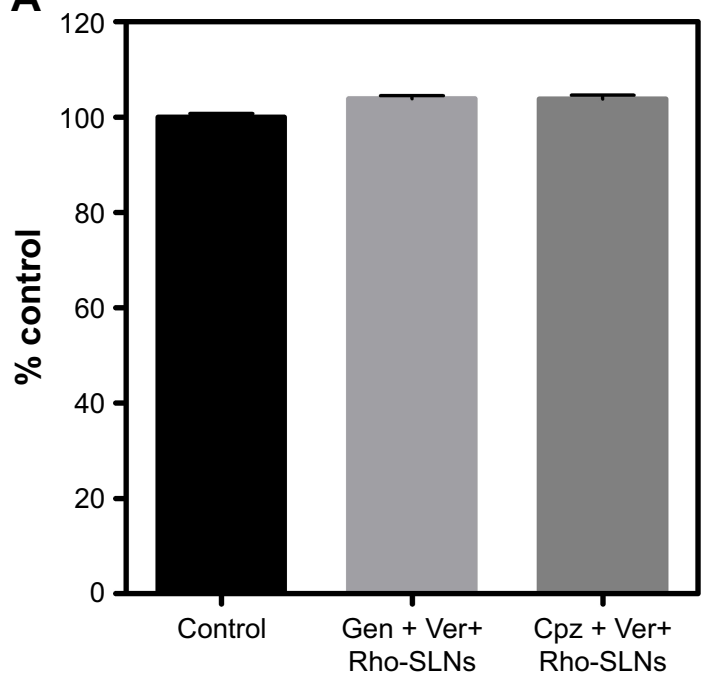

B

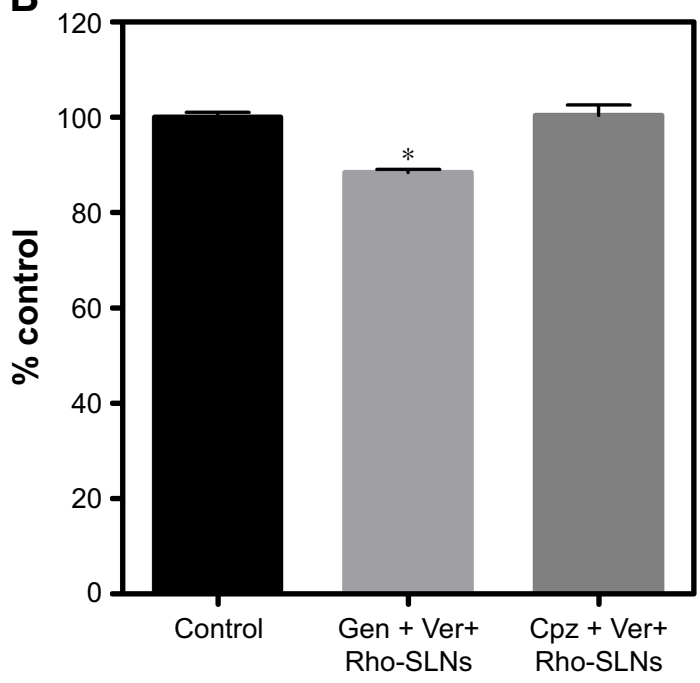

Figure 9 Effects of endocytosis inhibition by Gen and Cpz on uptake of Rho-SLNs in MCF7 (A) and MCF7/ADR (B) cells.

Notes: All cells treated concomitantly with Ver to eliminate the effect of Pgp-mediated efflux on Rho uptake. *Significantly different compared to endocytosis inhibitor in each cell line $(P<0.05)$.

Abbreviations: Gen, genistein; Cpz, chlorpromazine; Rho, rhodamine I23; SLNs, solid lipid nanoparticles; Ver, verapamil.

the internalization of nanoparticles based on size and surface properties. ${ }^{18,22,23}$ Endocytosis mechanisms are classified into pinocytosis and phagocytosis based on the size of the cargo, and then depending on the protein machinery involved, pinocytosis is divided into macropinocytosis, clathrin-mediated, caveola-mediated, and clathrin- and caveola-independent endocytosis. ${ }^{9,13,24}$ Different routes of endocytosis result in varied fates of cargos following internalization: nanoparticles internalized through phagocytosis, macropinocytosis, and clathrin-mediated endocytosis usually end up in lysosomes, while those going through caveola-mediated endocytosis can evade lysosomes, leading cargos to the nucleus. ${ }^{9,24}$ In the present study, SLNs did not show any superiority compared with unincorporated free form in intracellular delivery of cargo molecules, such as Ptx and Rho, into drug-sensitive MCF7 cells that did not express Pgp. However, SLNs delivered much more cargo molecules into MCF7/ADR cells, which are MDR cells expressing Pgp, suggesting that MCF7/ADR cells might
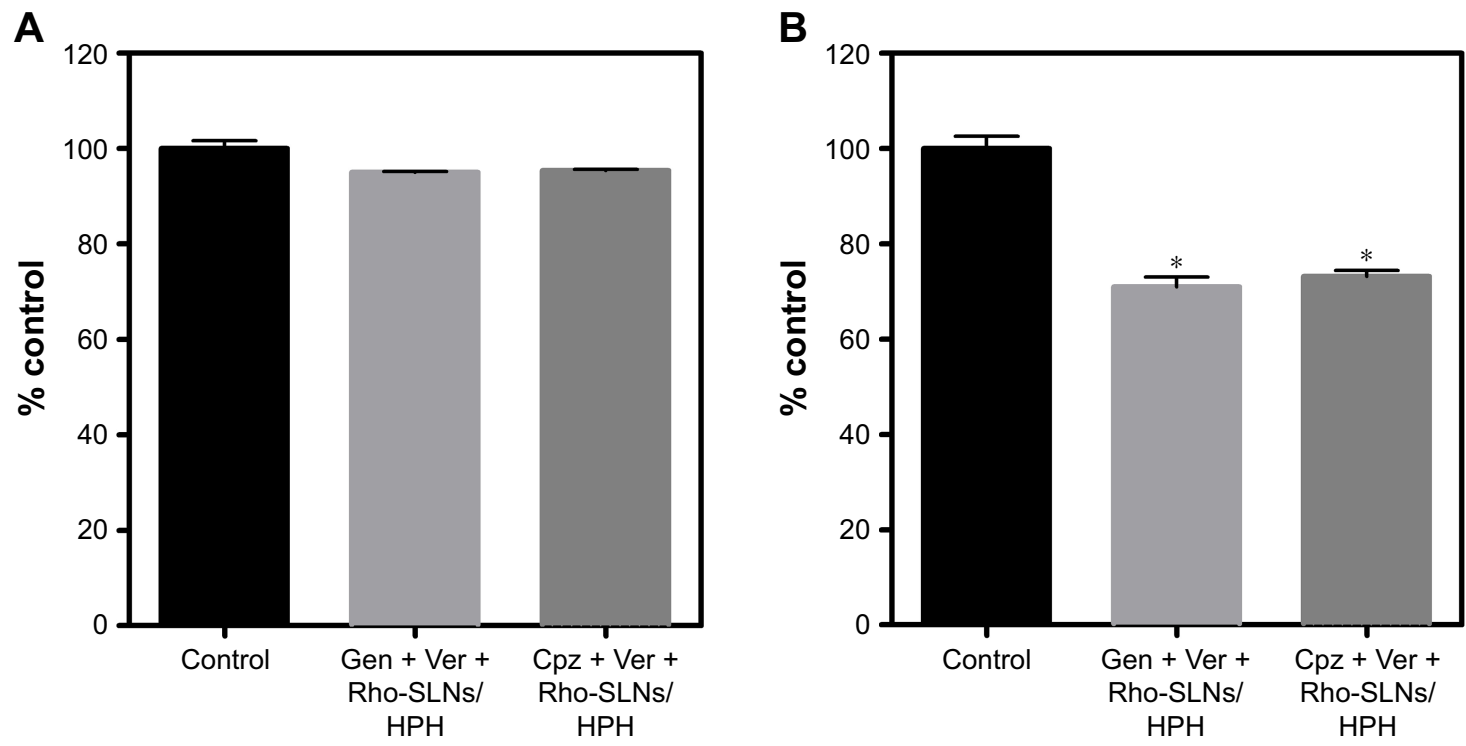

Figure 10 Effects of endocytosis inhibition by Gen and Cpz on Rho-SLN uptake with smaller mean particle size after HPH in MCF7 (A) and MCF7/ADR (B) cells. Notes: All cells were treated concomitantly with Ver to eliminate the effect of Pgp-mediated efflux on Rho uptake. *Significantly different compared to without endocytosis inhibitor in each cell line $(P<0.05)$.

Abbreviations: Gen, genistein; Cpz, chlorpromazine; Ver, verapamil; Rho, rhodamine I23; SLNs, solid lipid nanoparticles; HPH, high-pressure homogenization. 
A
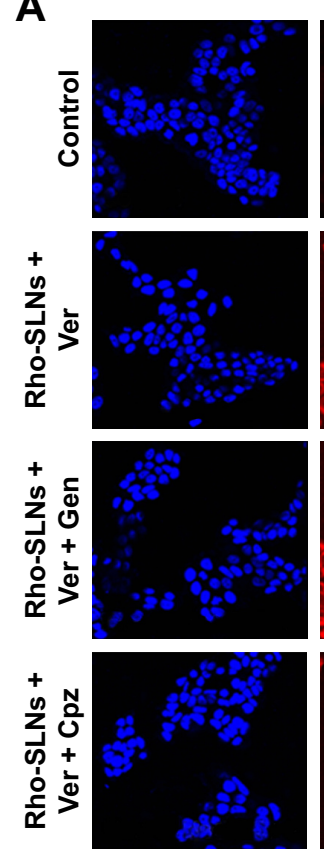
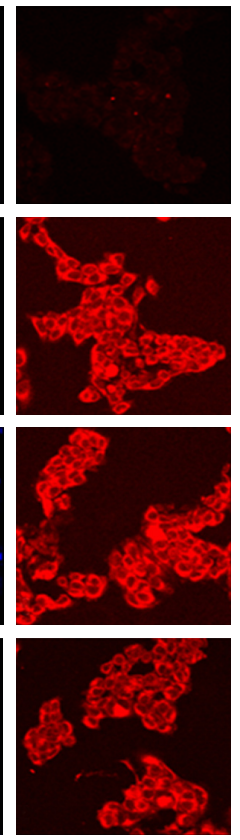
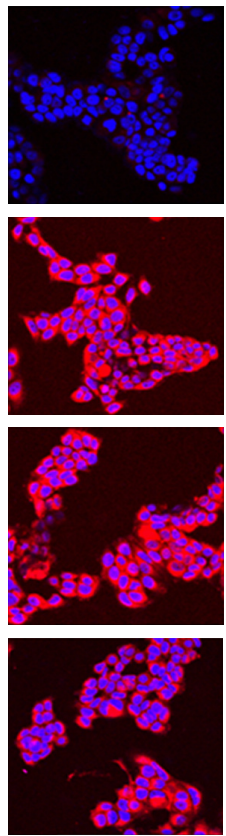

B
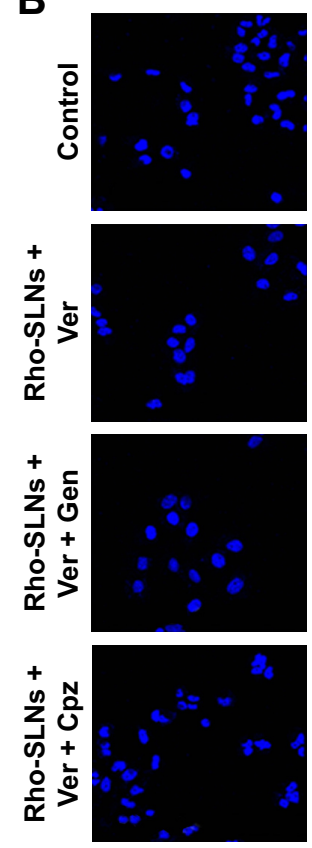
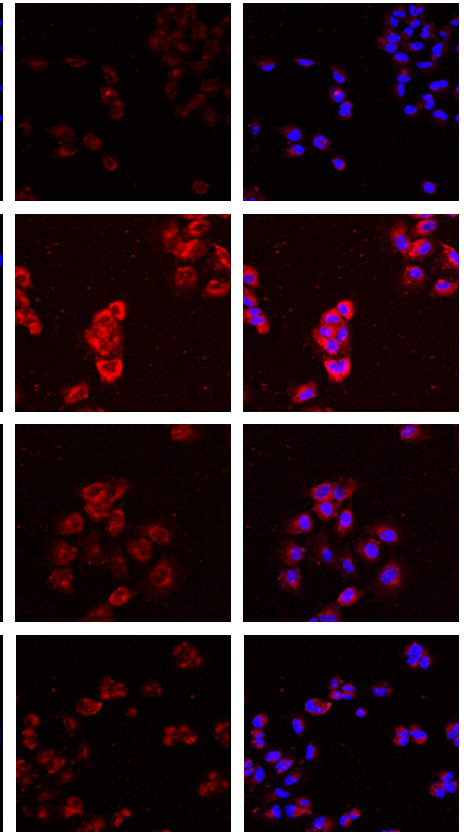

Figure I I Confocal laser-scanning microscopy micrographs of internalization of Rho-SLNs (red) in MCF7 (A) and MCF7/ADR (B) cells.

Notes: Endocytosis of cells was inhibited by $200 \mu \mathrm{M}$ Gen or $10 \mu \mathrm{g} / \mathrm{mL}$ Cpz. All cells were treated concomitantly with Ver to eliminate the effect of Pgp-mediated efflux on Rho uptake. Cell nuclei were stained with DAPI (blue).

Abbreviations: Rho, rhodamine 123; SLNs, solid lipid nanoparticles; Gen, genistein; Cpz, chlorpromazine; Ver, verapamil.

take up SLNs using different endocytosis pathways from those that MCF7 cells utilize. This hypothesis was supported by the distinctive expression patterns of caveolin 1 molecule between MCF7 and MCF7/ADR cells, as shown in Figure 6 . Both cell lines expressed clathrin, which is known as an essential component for constructing clathrin-coated pits in clathrin-mediated endocytosis. ${ }^{24}$ Unlike clathrin, caveolin 1 was detected only in MCF7/ADR cells, and not in MCF7 cells.
Caveolin 1 is known as one of the characteristic elements of caveolae that are small $(50-80 \mathrm{~nm})$ flask-shaped invaginations of plasma membrane formed in caveola-mediated endocytosis. ${ }^{25}$ Caveola-mediated endocytosis is probably the best-known clathrin-independent pathway, and unlike clathrin-coated pits, caveolae are present in most but not all types of cells. ${ }^{25}$ Each endocytosis route has been known to be used preferentially by certain macromolecules, eg, hTf has
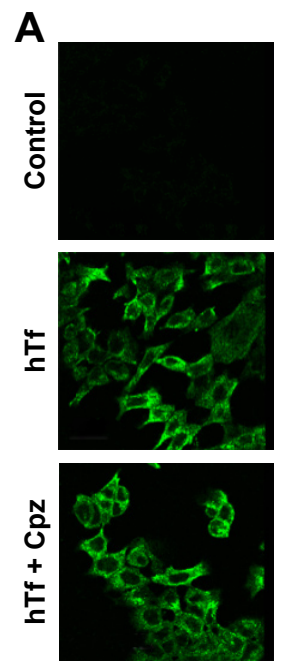
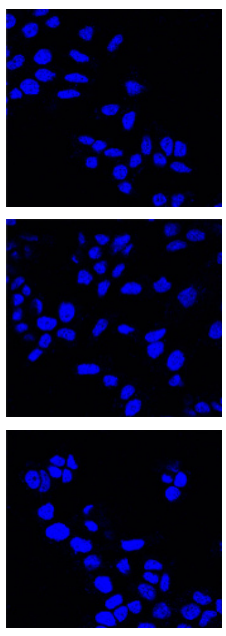
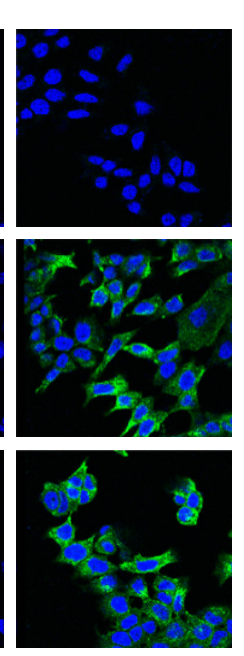

B
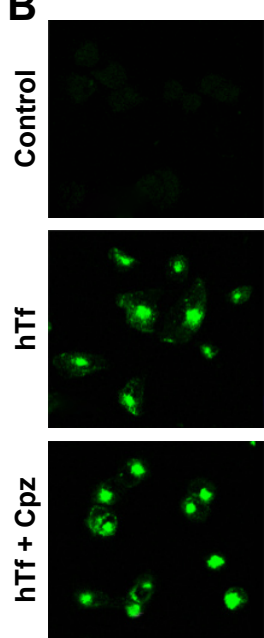
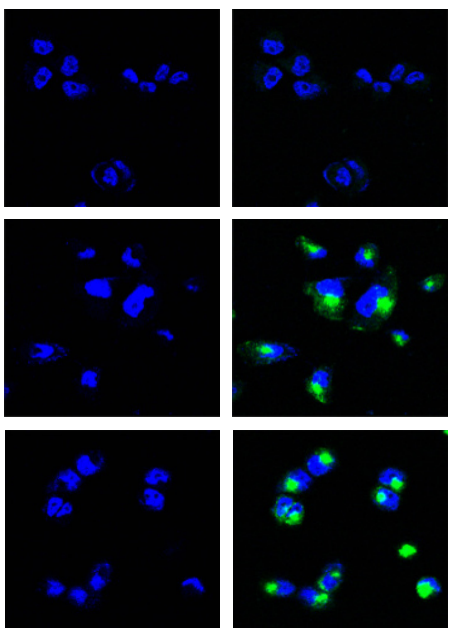

Figure 12 Effect of Cpz on intracellular uptake of hTf (green), known as a ligand for clathrin-mediated endocytosis in MCF7 (A) and MCF7/ADR (B) cells. Note: Cell nuclei were stained with DAPI (blue).

Abbreviations: Cpz, chlorpromazine; hTf, human serum transferrin. 
A
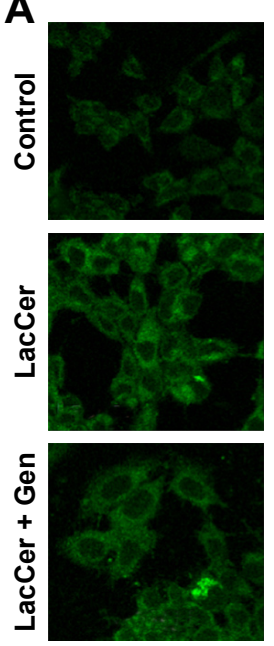
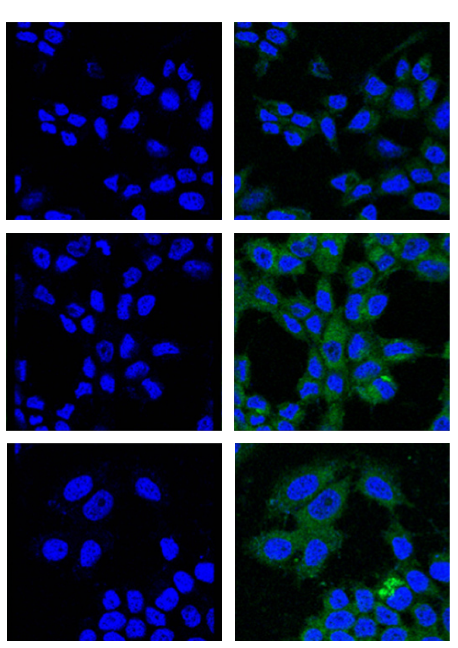

B
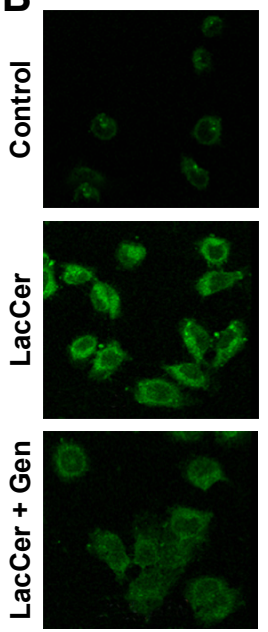
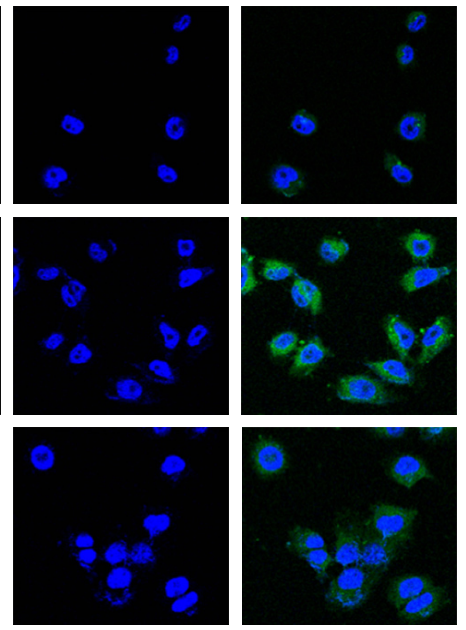

Figure 13 Effect of Gen on intracellular uptake of LacCer (green), known as a ligand for caveola-mediated endocytosis in MCF7 (A) and MCF7/ADR (B) cells. Note: Cell nuclei were stained with DAPI (blue).

Abbreviation: Gen, genistein.

been considered a representative marker of clathrin-mediated endocytosis, because it binds to Tf receptors in iron-bound form to be internalized by clathrin-mediated endocytosis. ${ }^{26,27}$ Meanwhile, LacCer has been used as a marker for caveolamediated endocytosis. ${ }^{28,29}$ LacCer is a glycosphingolipid that resides preferably in lipid rafts and is known to be internalized through clathrin-independent pathways that are dependent on dynamin and caveolin $1 .^{30-32}$ With specific endocytosis markers, endocytosis inhibitors have been used to reveal endocytosis mechanisms for various macromolecules and nanoparticles. $\mathrm{Cpz}$ is a cationic amphiphilic drug and known to inhibit the formation of clathrin-coated pits by a reversible translocation of clathrin and its adaptor proteins from plasma membrane to intracellular vesicles. ${ }^{33}$ Gen has been used to inhibit caveola-mediated endocytosis, because it causes disruption in the actin network at the site of endocytosis and blocks recruitment of dynamin II, both known as dispensable events in caveola-mediated endocytosis. ${ }^{34,35}$ In the present study, we tried to confirm that Gen and Cpz had no effect on Pgp pumps by measuring Rho efflux by Pgp in MCF7/ ADR cells. Unfortunately, Gen decreased Rho uptake by $22 \%$ in MCF7/ADR cells, suggesting the possibility that Gen induced Pgp in MCF7/ADR. However, Xue et al reported that Gen did not influence Pgp function and showed no effect on the expression of Pgp at both mRNA and protein levels at doses $\leq 330 \mu \mathrm{M}$ in MCF7/ADR cells. ${ }^{36}$ On the other hand, there have been studies that showed contradictory results where Gen increased Pgp expression and function at 1 and $10 \mu \mathrm{M}$ in the hepatocellular carcinoma-derived HepG2 cell line, ${ }^{37}$ and 14 days' administration of Gen reduced oral bioavailability of talinolol, a Pgp substrate, in healthy human participants. ${ }^{38}$ At any rate, Gen showed a modest induction of Pgp activity at $200 \mu \mathrm{M}$ in the present study, and this should be considered in the interpretation of the results obtained using Gen. In the case of $\mathrm{Cpz}$, Rho uptake was increased by $22 \%$, suggesting modest inhibition of Pgp by Cpz. Consistent results have been presented by other researchers using other cell lines, such as the Caco 2 intestinal epithelial cell line and LLC-PK1 and LMDR1 cells. ${ }^{39-41}$ However, the effect of Cpz was not as significant as Ver, a well-known Pgp inhibitor, which showed much greater increase of Rho accumulation in MCF7/ADR cells. In further studies, Ver was cotreated with Gen and $\mathrm{Cpz}$, which can cover up the effects of Gen and Cpz on Pgp. However, the moderate effects of Gen and Cpz on Pgp caused difficulties in the interpretation of Ptx-SLN uptake in MCF7/ADR cells, resulting in a slight reduction and increase in uptake by treatment with Gen and $\mathrm{Cpz}$, respectively, as shown in Figure 8. We expected the uptake reduction of Ptx-SLNs was due to the specific inhibition of caveola-mediated endocytosis by Gen. Unfortunately, the degree of decrease was not enough to distinguish the effects from Pgp induction and endocytosis inhibition. It was also similar in the case of Cpz. The increase in Ptx-SLN uptake was not clear enough to discern whether it was due to inhibition of clathrin-mediated endocytosis or inhibition of Pgp in addition to Ver.

In spite of all the doubts, the inhibition of Ptx-SLN uptake by Gen was likely due to suppression of caveola-mediated endocytosis because the effect on Pgp was covered up by Ver, and thus we concluded that caveola-mediated endocytosis was involved in Ptx-SLN and Rho-SLN uptake into MCF7/ ADR cells. 
In the experiment using Gen, it was predicted that SLN uptake into MCF7 cells would not be affected, because MCF7 cells did not express caveolin 1. In contrast, using $\mathrm{Cpz}$, we expected SLN uptake to be inhibited in both MCF7 and MCF7/ADR cells, because clathrin-mediated endocytosis is a common endocytosis route that is present and inherently active in all mammalian cells. ${ }^{24}$ However, in MCF7 cells, neither Gen nor Cpz caused any changes in Ptx-SLN or Rho-SLN uptake in our study. There was concern that broad particle-size distribution would hide the effects of endocytosis inhibitors, because nanoparticles might use multiple endocytosis routes depending on the particle size. To check this possibility, Rho-SLNs were prepared using HPH to obtain smaller particles with lower PI values (HPH RhoSLNs). Even with the smaller HPH Rho-SLNs, there was no change induced by Gen or $\mathrm{Cpz}$ in uptake into MCF7 cells, as shown in Figure 10A. Rejman et al showed that internalization of polystyrene latex particles of diameter $<200 \mathrm{~nm}$ into B16 cells involved clathrin-coated pits and increasing size shifted the endocytosis mechanism to the caveola-mediated pathway, which became the predominant one for particles $500 \mathrm{~nm}$ in size. ${ }^{28}$ It has been reported that Rho-loaded SLNs with mean size $<200 \mathrm{~nm}$ were internalized by human glioma cell lines through clathrin-mediated endocytosis. ${ }^{42}$ Although our HPH Rho-SLNs presented mean particle sizes $<200 \mathrm{~nm}$, which was expected to utilize clathrin-mediated endocytosis, Rho-SLNs/HPH likely used clathrin-independent routes, judging from no change in uptake by $\mathrm{Cpz}$ in MCF7 cells. Further studies will be required to examine the internalization mechanisms involved in MCF7 cells fully.

In contrast to MCF7 cells, caveola-mediated pathways appeared to be involved in the uptake of Ptx-SLNs into MCF7/ADR cells, based on the modest but statistically significant reduction in uptake by Gen, even though the contribution of the caveola-mediated process did not seem to be strong. In the same manner as uptake into MCF7 cells, uptake of Ptx-SLNs into MCF7/ADR cells was not affected by $\mathrm{Cpz}$, suggesting that mechanisms other than clathrinmediated endocytosis were involved in Ptx-SLN internalization. Likewise, uptake of Rho-SLNs of similar particle size and PI values to Ptx-SLNs was affected by Gen and Cpz. As mentioned, a size effect could not be excluded for switching endocytosis mechanisms in both cell lines, which prompted us to compare the uptake of Rho-SLNs $(223.6 \pm 138.2 \mathrm{~nm})$ with that of HPH Rho-SLNs $(150.8 \pm 0.2 \mathrm{~nm})$. Surprisingly, the reduction in HPH Rho-SLN uptake by both Gen and $\mathrm{Cpz}$ in MCF7/ADR cells was more noticeable than for
Rho-SLNs, indicating that decreasing particle size switched the internalization mechanism from clathrin-independent endocytosis to a clathrin-dependent one. In addition, the contribution of caveola-mediated endocytosis appeared to be augmented in MCF7/ADR cells by reducing the particle size of SLNs. Size-dependent switching to clathrin-mediated endocytosis was consistent with another study in which the researchers proposed that internalization of microspheres of diameter $<200 \mathrm{~nm}$ involved clathrin-mediated endocytosis and increasing size shifted to caveola-mediated endocytosis, which became the predominant pathway for particles $500 \mathrm{~nm}$ in size. ${ }^{28}$

Unlike MCF7/ADR cells, there were still no changes observed with Gen or Cpz in MCF7 cells for HPH Rho-SLN uptake, which proposed that even smaller SLNs did not utilize clathrin-mediated endocytosis in MCF7 cells. On the other hand, there was a possibility that $\mathrm{Cpz}$ was not a specific inhibitor for clathrin-mediated endocytosis, as proposed in another study that showed poor specificity of Gen and Cpz for inhibiting distinctive endocytic pathways by analyzing the uptake of LacCer and hTf. ${ }^{43}$ The effects of Gen and Cpz on the uptake of corresponding markers, such as LacCer and hTf, were cell-dependent, which prompted us to test the specificity of Gen and $\mathrm{Cpz}$ in MCF7 and MCF7/ADR. ${ }^{43}$ Confocal images for LacCer and hTf uptake showed that the inhibitory effects of both Gen and Cpz were weak in MCF7 cells, but more clearly observed in MCF7/ADR cells. Due to poor specificity, further study will be required to reveal precise mechanisms utilized by SLNs in MCF7 cells. However, in spite of the pitfalls of inhibitors, it was clearly shown that MCF7 and MCF7/ADR used different endocytosis mechanisms to take up SLNs in the present study.

\section{Conclusion}

Ptx-SLNs showed remarkably enhanced anticancer activity in MCF7/ADR compared to Ptx delivered in DMSO and Cre-based vehicles. SLNs significantly increased intracellular Ptx and Rho uptake. Western blotting demonstrated that clathrin was expressed in both cell lines, while caveolin 1 was expressed only in MCF7/ADR. In MCF7/ADR, PtxSLN and Rho-SLN uptake was reduced by Gen, with no change by $\mathrm{Cpz}$, proposing involvement of caveola-mediated endocytosis. Size reduction of Rho-SLNs by HPH appeared to cause a shift of endocytosis mechanism from a clathrinindependent pathway to clathrin-dependent one. In contrast to MCF7/ADR, the uptake of SLNs into MCF7 was not changed by Gen or Cpz, suggesting involvement of clathrinand caveola-independent mechanisms for the entry of SLNs. 
In conclusion, MDR was reversed by incorporating drug into SLNs and the reversal mediated by increased SLN uptake, evading efflux pumps in MDR cells. The enhanced uptake could also have been due to the use of different endocytosis pathways by SLNs in MDR cells from drug-sensitive cancer cells.

\section{Acknowledgment}

This study was supported by Woosuk University.

\section{Disclosure}

The authors report no conflicts of interest in this work.

\section{References}

1. Payne KK, Manjili MH. Adaptive immune responses associated with breast cancer relapse. Arch Immunol Ther Exp (Warsz). 2012;60(5):345-350.

2. Joerger M, Thürlimann B. Chemotherapy regimens in early breast cancer: major controversies and future outlook. Expert Rev Anticancer Ther. 2013;13(2):165-178.

3. Redden MH, Fuhrman GM. Neoadjuvant chemotherapy in the treatment of breast cancer. Surg Clin North Am. 2013;93(2):493-499.

4. Fuksa L, Micuda S, Grim J, Ryska A, Hornychova H. Predictive biomarkers in breast cancer: their value in neoadjuvant chemotherapy. Cancer Invest. 2012;30(9):663-678.

5. Peetla C, Vijayaraghavalu S, Labhasetwar V. Biophysics of cell membrane lipids in cancer drug resistance: Implications for drug transport and drug delivery with nanoparticles. Adv Drug Deliv Rev. 2013; 65(13-14):1686-1698.

6. Aller SG, Yu J, Ward A, et al. Structure of P-glycoprotein reveals a molecular basis for poly-specific drug binding. Science. 2009;323(5922): 1718-1722.

7. Cohen AW, Hnasko R, Schubert W, Lisanti MP. Role of caveolae and caveolins in health and disease. Physiol Rev. 2004;84(4):1341-1379.

8. Thomas H, Coley HM. Overcoming multidrug resistance in cancer: an update on the clinical strategy of inhibiting p-glycoprotein. Cancer Control. 2003;10(2):159-165.

9. Singh MS, Tammam SN, Shetab Boushehri MA, Lamprecht A. MDR in cancer: Addressing the underlying cellular alterations with the use of nanocarriers. Pharmacol Res. 2017;126:2-30.

10. Choi YH, Han HK. Nanomedicines: current status and future perspectives in aspect of drug delivery and pharmacokinetics. J Pharm Investig. 2018;48(1):43-60.

11. Youn YS, Kwag DS, Lee ES. Multifunctional nano-sized fullerenes for advanced tumor therapy. J Pharm Investig. 2017;47(1):1-10.

12. Sarisozen C, Pan J, Dutta I, Torchilin VP. Polymers in the co-delivery of siRNA and anticancer drugs to treat multidrug-resistant tumors. J Pharm Investig. 2017;47(1):37-49.

13. Sahay G, Alakhova DY, Kabanov AV. Endocytosis of nanomedicines. $J$ Control Release. 2010;145(3):182-195.

14. de Weger VA, Beijnen JH, Schellens JH. Cellular and clinical pharmacology of the taxanes docetaxel and paclitaxel - a review. Anticancer Drugs. 2014;25(5):488-494.

15. Lee MK, Lim SJ, Kim CK. Preparation, characterization and in vitro cytotoxicity of paclitaxel-loaded sterically stabilized solid lipid nanoparticles. Biomaterials. 2007;28(12):2137-2146.

16. Sosnik A. Reversal of multidrug resistance by the inhibition of ATPbinding cassette pumps employing "Generally Recognized As Safe" (GRAS) nanopharmaceuticals: a review. Adv Drug Deliv Rev. 2013; 65(13-14):1828-1851.

17. Baek JS, Cho CW. Controlled release and reversal of multidrug resistance by co-encapsulation of paclitaxel and verapamil in solid lipid nanoparticles. Int J Pharm. 2015;478(2):617-624.
18. Gao Z, Zhang L, Sun Y. Nanotechnology applied to overcome tumor drug resistance. J Control Release. 2012;162(1):45-55.

19. Kunjachan S, Błauż A, Möckel D, et al. Overcoming cellular multidrug resistance using classical nanomedicine formulations. Eur J Pharm Sci. 2012;45(4):421-428.

20. Li W, Zhang H, Assaraf YG, et al. Overcoming ABC transportermediated multidrug resistance: Molecular mechanisms and novel therapeutic drug strategies. Drug Resist Updat. 2016;27:14-29.

21. Miao J, Du YZ, Yuan H, Zhang XG, Hu FQ. Drug resistance reversal activity of anticancer drug loaded solid lipid nanoparticles in multidrug resistant cancer cells. Colloids Surf B Biointerfaces. 2013; 110:74-80.

22. Oh N, Park JH. Endocytosis and exocytosis of nanoparticles in mammalian cells. Int J Nanomedicine. 2014;9(Suppl 1):51-63.

23. Patiño T, Soriano J, Barrios L, Ibáñez E, Nogués C. Surface modification of microparticles causes differential uptake responses in normal and tumoral human breast epithelial cells. Sci Rep. 2015;5:11371.

24. Doherty GJ, McMahon HT. Mechanisms of endocytosis. Annu Rev Biochem. 2009;78:857-902.

25. Parton RG, del Pozo MA. Caveolae as plasma membrane sensors, protectors and organizers. Nat Rev Mol Cell Biol. 2013;14(2):98-112.

26. Widera A, Norouziyan F, Shen WC. Mechanisms of TfR-mediated transcytosis and sorting in epithelial cells and applications toward drug delivery. Adv Drug Deliv Rev. 2003;55(11):1439-1466.

27. Maxfield FR, McGraw TE. Endocytic recycling. Nat Rev Mol Cell Biol. 2004;5(2):121-132.

28. Rejman J, Oberle V, Zuhorn IS, Hoekstra D. Size-dependent internalization of particles via the pathways of clathrin- and caveolae-mediated endocytosis. Biochem J. 2004;377(Pt 1):159-169.

29. Zuhorn IS, Kalicharan R, Hoekstra D. Lipoplex-mediated transfection of mammalian cells occurs through the cholesterol-dependent clathrin-mediated pathway of endocytosis. J Biol Chem. 2002;277(20): 18021-18028.

30. Puri V, Watanabe R, Singh RD, et al. Clathrin-dependent and independent internalization of plasma membrane sphingolipids initiates two Golgi targeting pathways. J Cell Biol. 2001;154(3):535-547.

31. Singh RD, Puri V, Valiyaveettil JT, Marks DL, Bittman R, Pagano RE. Selective caveolin-1-dependent endocytosis of glycosphingolipids. Mol Biol Cell. 2003;14(8):3254-3265.

32. Marks DL, Singh RD, Choudhury A, Wheatley CL, Pagano RE. Use of fluorescent sphingolipid analogs to study lipid transport along the endocytic pathway. Methods. 2005;36(2):186-195.

33. Wang LH, Rothberg KG, Anderson RG. Mis-assembly of clathrin lattices on endosomes reveals a regulatory switch for coated pit formation. J Cell Biol. 1993;123(5):1107-1117.

34. Nabi IR, Le PU. Caveolae/raft-dependent endocytosis. J Cell Biol. 2003;161(4):673-677.

35. Parton RG, Joggerst B, Simons K. Regulated internalization of caveolae. J Cell Biol. 1994;127(5):1199-1215.

36. Xue JP, Wang G, Zhao ZB, Wang Q, Shi Y. Synergistic cytotoxic effect of genistein and doxorubicin on drug-resistant human breast cancer MCF-7/Adr cells. Oncol Rep. 2014;32(4):1647-1653.

37. Rigalli JP, Ciriaci N, Arias A, et al. Regulation of multidrug resistance proteins by genistein in a hepatocarcinoma cell line: impact on sorafenib cytotoxicity. PLoS One. 2015;10(3):e0119502.

38. Xiao CQ, Chen R, Lin J, et al. Effect of genistein on the activities of cytochrome P450 3A and P-glycoprotein in Chinese healthy participants. Xenobiotica. 2012;42(2):173-178.

39. Brown D, Goosen TC, Chetty M, Hamman JH. Effect of oral contraceptives on the transport of chlorpromazine across the CACO-2 intestinal epithelial cell line. Eur J Pharm Biopharm. 2003;56(2):159-165.

40. Wang JS, Zhu HJ, Markowitz JS, Donovan JL, DeVane CL. Evaluation of antipsychotic drugs as inhibitors of multidrug resistance transporter P-glycoprotein. Psychopharmacology (Berl). 2006;187(4):415-423.

41. Boulton DW, DeVane CL, Liston HL, Markowitz JS. In vitro P-glycoprotein affinity for atypical and conventional antipsychotics. Life Sci. 2002;71(2):163-169. 
42. Martins S, Costa-Lima S, Carneiro T, Cordeiro-da-Silva A, Souto EB, Ferreira DC. Solid lipid nanoparticles as intracellular drug transporters: an investigation of the uptake mechanism and pathway. Int J Pharm. 2012;430(1-2):216-227.
43. Vercauteren D, Vandenbroucke RE, Jones AT, et al. The use of inhibitors to study endocytic pathways of gene carriers: optimization and pitfalls. Mol Ther. 2010;18(3):561-569.

\section{Publish your work in this journal}

The International Journal of Nanomedicine is an international, peerreviewed journal focusing on the application of nanotechnology in diagnostics, therapeutics, and drug delivery systems throughou the biomedical field. This journal is indexed on PubMed Central, MedLine, CAS, SciSearch $\AA$, Current Contents ${ }^{\circledR} /$ Clinical Medicine,
Journal Citation Reports/Science Edition, EMBase, Scopus and the Elsevier Bibliographic databases. The manuscript management system is completely online and includes a very quick and fair peer-review system, which is all easy to use. Visit http://www.dovepress.com/ testimonials.php to read real quotes from published authors.

Submit your manuscript here: http://www.dovepress.com/international-journal-of-nanomedicine-journal 